\title{
Association Between Serum Procalcitonin Levels and Disseminated Intravascular Coagulation in Patients With Septic Shock
}

\section{Guang Fu}

Shenzhen Second People's Hospital

Xi-si He

Shenzhen Second People's Hospital

\section{Xiao-peng Cao}

4th Hospital of PLA

\section{Hao-li Li}

Shenzhen University First Affiliated Hospital: Shenzhen Second People's Hospital

\section{Hai-chao Zhan}

Shenzhen Second People's Hospital

Jun-fu Lu

Shenzhen Second People's Hospital

\section{Yan-hong Chen}

Shenzhen University First Affiliated Hospital: Shenzhen Second People's Hospital

\section{Zi-Long Yang}

Shenzhen Second People's Hospital

Jia-jia Huang

Shenzhen Second People's Hospital

\section{Zhi-peng Zhou}

Shenzhen Second People's Hospital Department of Neurology

\section{Yong-wen Feng}

Shenzhen Second People's Hospital

\section{Ying-yi Luan}

4th Hospital of PLA

\section{Ming Wu ( $\sim$ boshiyy@126.com )}

Department of Critical Care Medicine and Hospital Infection Prevention and Control, The Second People's Hospital of Shenzhen \& First Affiliated Hospital of Shenzhen University, Health Science Center, Shenzhen, China https://orcid.org/0000-0002-0800-5506

\section{Research Articles}


Keywords: Procalcitonin, Septic Shock, Disseminated Intravascular Coagulation

Posted Date: January 4th, 2021

DOI: https://doi.org/10.21203/rs.3.rs-136390/v1

License: (c) (1) This work is licensed under a Creative Commons Attribution 4.0 International License. Read Full License 


\section{Abstract}

Objectives: To investigated the relationships between procalcitonin (PCT) and disseminated intravascular coagulation (DIC) during septic shock.

Methods: A retrospective study was performed, which included septic shock patients admitted into intensive care unit (ICU) from January 1, 2015 to December 31, 2018.

DIC was defined as international society of thrombosis and homeostasis criteria (ISTH $\geq 5$ ). PCT was based on the first value after admission into ICU and the routine biochemical coagulation data based on the worst value extracted from electronic medical records within 24 hours on admission into ICU.

Results: Among 2156 patients screened, 164 patients with septic shock were included in the finally analysis and $35.4 \%$ (58/164) of whom developed DIC after admission. PCT level was significantly higher in septic shock patients who developed DIC than those who did not (54.6[13.6-200] vs12.6[2.4-53.3] $\mathrm{ng} / \mathrm{ml}, \mathrm{P}<0.001)$. Multivariable logistic regression revealed that PCT $(\mathrm{OR}=1.011,95 \% \mathrm{Cl} 1.006-1.016$, $P<0.001)$ was associated with DIC during septic shock. Curve fitting showed a positive correlation between PCT and DIC. The Receiver Operating characteristic (ROC) curve suggested that the cut-off point for PCT to predict DIC during septic shock was $42.1 \mathrm{ng} / \mathrm{ml}$, with sensitivity $60.34 \%$, specificity $72.74 \%$ and the area under the curve (AUC) $0.701(95 \% \mathrm{Cl}[0.619-0.784], \mathrm{P}<0.001)$. Interestingly, PCT increased early detection of DIC during septic shock compared with other risk factors $(P=0.012)$

Conclusions: Our data suggest that PCT level over $42.1 \mathrm{ng} / \mathrm{ml}$ on admission is associated with DIC during septic shock, and PCT is a potential predictive factor of DIC induced by septic shock at early stage.

\section{Introduction}

Activation of coagulation system by multiple factors in sepsis, in addition to immune response, is recognized as a host defense strategy against infection, which is termed as immunothrombosis. $(1,2)$ However, excessive and dysregulated activation of coagulation often leads to very severe coagulopathy characterized by massive formation of thrombi, particularly, in microcirculation, which is termed as disseminated intravascular coagulation (DIC). (3) DIC is a commonly encountered life-threatening complication in $30-50 \%$ sepsis patients with mortality up to $43 \% .(4,5)$ The occurrence of DIC is often characterized by systemic activation of coagulation, bleeding and subsequent organ dysfunctions. (6) Additionally, DIC has been identified as a potent indicator of multiple organ dysfunction syndrome(MODS) and mortality in severe septic patients. (7) Treatment for DIC using anticoagulants at early stage has shown great potential to improve the patient's outcomes. (8) Thus, early detection and initiation of preventive strategies indicates tremendous clinical significance.

Conventionally, early diagnosis of DIC in septic patients largely depends on the established over-DIC diagnostic criteria released by International Society of Thrombosis and Homeostasis (ISTH). (6) The essential components of this criteria include platelet counts, D-dimer, Prothrombin time, Fibrinogen, 
System Inflammatory Reaction Syndrome (SIRS) score, sequential organ failure assessment(SOFA) score. (6) Other three score systems have also been proposed. (9-11) However, these current clinical score systems are generally similar based on routine tests and often failed to detect early stage of DIC prior to apparent onsets of coagulopathy (12). Additionally, various other aspects of sepsis and coagulopathy are not covered within those components. And studies have shown that other parameters (such as APACHEll score, $(13,14)$ serum lactate, $(15)$ and proinflammatory cytokines $(16,17)$ ) are also associated DIC development. Hence, the value of those factors to predict the development of DIC in septic shock patients warrants further investigation.

Procalcitonin (PCT) is the peptide precursor of the hormone calcitonin and its plasma level is expected to be very low in normal conditions.(18) However, PCT is dramatically produced and ubiquitously released with 1000-fold elevation after infection. (19) Thus, it has been used as a valuable biomarker of ongoing bacterium infection and sepsis diagnosis. (20) Moreover, Inflammation and coagulopathy are always interconnected in septic shock.(21) Early biomarkers of clotting activation (thrombinantithrombin complex (TAT), a2-plasmininhibitor-plasmin complex (PIC), soluble thrombomodulin (sTM), and tissue plasminogen activator-inhibitor complex (tPAIC)) are not routinely available in developing countries. Therefore, the coagulation disorder, especially DIC induced by septic shock, cannot be readily detected at early stage. As a sensitive biomarker of inflammation, PCT level is routinely tested in ICU. Therefore, we hypothesized that PCT level at admission into ICU may offer great value in detection of DIC at early stage in septic shock patientadmitted to adult ICU.

\section{Methods}

\section{Design, Setting, and population}

This retrospective study was designed by the investigators and performed at the Second People's Hospital of Shenzhen from January 12015 to December 31 2018. Data were obtained from electronic medical records of patients with septic shock hospitalized at department of critical care medicine during the study dates. The following inclusion criteria were used: septic shock 3.0 criteria (a) Persisting hypotension requiring vasopressors to maintain mean arterial pressure (MAP) $\geq 65 \mathrm{mmHg}$; (b) Blood lactate $>2 \mathrm{mmol} / \mathrm{L}$ despite adequate volume resuscitation; (22) Excluding criteria were listed following: (a) Age <18years old; (b) cardio-pulmonary resuscitation; (c) History of thrombotic diseases and rheumatic or immune or hematological diseases or tumors; (d)The length of ICU stay is less than 24 hours;(e)cases with missing data (No PT, APTT or INR were found within 24 hours after admission)(Figure 1).

\section{Clinical variables and definition}

All routinely collected vital signs and symptoms and laboratory values were extracted from the electronic medical records retrospectively. Data included, but were not limited to, demographic data (e.g. age, gender), biochemical parameters (e.g. blood cell count, liver function, kidney function, coagulation function, blood gas analysis), mechanic ventilation and infection sources. Procalcitonin was obtained with immunochemiluminometric assay and was defined as first value in ICU after admission. The worst 
value of other biochemical parameters within 24 hours admission to ICU was adopted for downstream analysis. We calculated the Acute Physiology and Chronic Health Evaluation (APACHE) II score within first 24 hours of hospitalization, and DIC was defined as ISTH scores $\geq 5$ within 24 hours on admission according to ISTH criteria. (6)

\section{Statistical analysis}

Quantitative parameters are presented as the means \pm standard deviations or medians and interquartile ranges (25th, 75th percentiles), and qualitative parameters are expressed as numbers and percentages. Continuous variables were compared using the independent two-sample t-test or Mann-Whitney U-test. Categorical variables were compared using the chi square test or Fisher's exact test. Univariate logistic regression analysis was performed to evaluate risk factors associated with DIC. All variables with $\mathrm{P}<0.1$ in univariate analysis were entered into a multivariate logistic regression with crude model and fully adjusted model: OR (odds ratioखand $95 \%$ confidence interval levels $(95 \% \mathrm{Cl})$. The predictive ability of PCT and other parameters for DIC was assessed using the AU-ROC curve method with MedCalc. The optimal cutoff value was determined using Youden's index. Then, we explored the relationship between PCT and DIC by smooth curve fitting after adjustment for potential confounders. Statistical analysis was performed using the statistical package SAS 9.4 (Windows, SAS Institute, Cary, North Carolina). P value (two-tailed) below 0.05 was considered statistically significant.

\section{Results}

\section{Basic characteristics of study population}

A total of 2154 patients were admitted to our ICU from January 1st 2015 to December 31st, 2018 including 261 (12.1\%) septic shock patients by the definition of Sepsis 3.0 (22). Only 164 patients were eventually enrolled in our study based on including and excluding criteria up tofollow-up discharge (Fig.1). The general characteristics of those patients with or without DIC were shown in Table 1. Among the total 164 patients, 58(35.4\%) patients developed DIC on admission in 24hours. And the 28-day mortality of DIC and non-DIC patients was $53.4 \%$ and $38.7 \%$, respectively. Patients who developed DIC had elevated lactate level, longer length of stay in ICU and higher APACHEll score, worse liver function (higher alanine transaminase(ALT), aspartate aminotransferase (AST) and total bilirubin) and kidney function (higher creatinine and blood urea nitrogen), and significant higher PCT level ( $54.6 \mathrm{ng} / \mathrm{ml}$ vs 12.6 $\mathrm{ng} / \mathrm{ml}, \mathrm{P}<0.001)$ as well as higher ISTH score $(5.0 \mathrm{vs} 30, \mathrm{P}<0.001)$, compared to those who did not develop DIC. Moreover, PCT was positively associated with ISTH score in our study (Fig.2). Notably, age and gender did not seem to affect the development of DIC. And no difference of blood leukocyte number was observed between those two groups.

\section{Risk factors associated with DIC induced by septic shock}

To explore the risk factors associated with DIC induced by septic shock, we choose all parameters and APACHE-II score as variables. As the results shown in Table 2, only PCT, total bilirubin, lactate and 
APACHEIl score were identified as significant risk factors to development of DIC in septic shock patients in univariant logistic regression model. After adjustment age, gender for multivariant logistic regression model, only PCT, total bilirubin and lactate were still statistically significant risk factors to development of DIC in septic shock patients. (Table 2).

\section{PCT improves the prediction of DIC induced by septic shock}

To explore other risk factors to predict DIC development subsequently with septic shock patients. ROC curve analysis was performed individually and combinatorically (Table3 and Fig.3A). The AUC for PCT was $0.701(95 \% \mathrm{Cl}[0.619-0.784], \mathrm{P}<0.001)$ and was significantly greater than those for total bilirubin, APACHII score and lactate $(P=0.01,0.002,<0.001)$ respectively. The optimal cut-off point for PCT for predicting DIC induced by septic shock was $42.1 \mathrm{ng} / \mathrm{ml}$, with a sensitivity of $60.34 \%$, specificity of $79.64 \%$. Then, prediction models incorporating clinical and biomedical risk factors were further analyzed. When using combination of lactate, total bilirubin and APACHII score to predict the DIC development in septic shock patients, the AUC was $0.706(95 \% \mathrm{Cl}, 0.630-0.774)$, Introducing PCT into this model statistically significantly increased the AUC to 0.801 ( $95 \% \mathrm{Cl} 0.732-0.859$; $\mathrm{P}=0.012$ ) (Table3 and Fig.3B), which indicated that PCT could significantly improve the prediction of DIC in septic shock patients.

\section{Discussion}

This retrospective study aimed to investigate the potential relationship between PCT and DIC. we found that PCT, lactate and total bilirubin at admission of ICU were independently associated with septic shock induced DIC development. Interestingly, incorporation of procalcitonin (inflammation biomarker) with lactate, total bilirubin and APACHII score improved the performance of predictive model of DIC development in septic shock patients. Our finding may help to provide a screening tool to detect DIC development at early stage.

PCT was reported as a biomarker of infection and sepsis and higher level of PCT was associated with higher serum proinflammatory cytokines. $(16,20)$ and such proinflammatory mediators could potently contribute to occurrence of coagulopathy through various mechanisms.(23) Significantly higher PCT level was also observed in septic patients with DIC compared to those without. $(16,24)$ As we known, during microorganism infection, inflammation and coagulation are involved in the development of septic complications, and mainly characterized by inflammation in early stage and obvious coagulation disorders in middle or the late stage. Thus, the early inflammatory indicators (PCT at ICU admission) may be helpful to predict the later severe coagulopathy(overt DIC, ISTH $\geq 5$ ). Our study confirmed this speculation by showing a positive correlation between PCT at admission and DIC development in septic shock patients. Moreover, our data may help to potentially establish a potential screening tool for physicians to assess the chances of DIC development at early stage following septic shock.

As expected, we observed a significantly higher PCT level in septic shock patients with DIC $(54.6 \mathrm{ng} / \mathrm{ml})$ than those without DIC $(12.6 \mathrm{ng} / \mathrm{ml})$. Hoppensteadt D reported a similar observation of higher PCT level in DIC patients despite of the numerical discrepancy, where they reported that PCT was $11.2 \mathrm{ng} / \mathrm{ml}$ in overt 
DIC group and 5.3ng/ml in non-overt group.(24) This discrepancy could be explained by different study participants. While they included patients with sepsis, we were focused on septic shock patients, whose severity of the disease, as well as PCT level, was far more than that of sepsis patients.

Notably, more than 30 million people are affected by sepsis worldwide and the incidence has been increasing over the past several decades, though the mortality has declined significantly with the advancement of diagnosis and management of sepsis. (25-27) One of the most frequently encountered complications is the occurrence of DIC, which is the hallmark of the failure of hemostatic system. (28) Currently, there are three guidelines for diagnosis and treatment of DIC that have been published. (9-11) Although those three guidelines are generally similar, they have some key differences. Therefore, the ISTH integrated those three guidelines and published its own guidance of diagnosis and treatment for DIC.(29) which has been accepted as the international standard and was used in this study. Moreover, ISTH overtDIC score has also been used to screen DIC on ICU day 1, which was shown to be associated significantly decreased mortality in a multicenter retrospective cohort. (30) However, no single parameter has been reported to effectively predict DIC in septic shock patients. Previous study have shown that plasma biomarkers, including TAT, PIC, sTM, and tPAIC, demonstrated good diagnostic performance and prognostic value in DIC patients with different underlying diseases. Besides, TAT, TPAIC and STM have certain implications in pre-DIC stage. (31) Combination of those four makers was demonstrated better prediction accuracy than single one alone. But the early biomarkers of clotting activation (TAT, PIC, etc.) are not routinely available in developing countries. Therefore, as an easily available inflammatory marker in clinical practice, PCT can play an important warning role in septic shock-related pre-DIC in developing countries.

Lactate is an anerobic metabolite that is commonly used as a marker for the function of microcirculation. During the early development of DIC, the excessive formation of microthrombi occludes the blood supply and causes tissue hypoxia, then eventually results in dysfunction of microcirculation and elevation of lactate level. Additionally, another study has shown that increased lactate level could predict the 90-day mortality of septic patients with DIC.(15) Thus, lactate level elevation may reflect the early stage of DIC development, as evidenced by our finding that lactate level was an independent risk factor for DIC.

Bilirubin is a biochemical indicator of liver function. Hyperbilirubinemia(hepatic dysfunction) is not uncommonly observed in patients with severe sepsis.(32) However, literatures on the study of hyperbilirubinemia in sepsis were very limited. Data form one retrospective study indicated that elevated serum bilirubin levels within 72 hours of admission were associated increased mortality in patients with severe sepsis and septic shock. (33) In our study, we identified serum bilirubin as an independent risk factor for development of DIC in septic shock patients. The mechanisms of this correlation are multifaceted and warrant further investigation. One plausible explanation would be that elevated serum bilirubin is a marker of liver dysfunction which is aggravated by DIC development.

Several aspects of limitations present in our study should be noted. Firstly, this is a retrospective study based on a small population at single center. Secondly, although we tried to adjust the potential bias, 
other unknown factors may also confound our results. Thirdly, this is a preliminary study to explore the potential association between PCT and DIC development in septic shock patients and large multicenter prospective studies are needed to confirm our findings.

In conclusion, our study revealed an unappreciated clinical value of PCT in prediction of DIC development in septic shock patients. Better predictive performance could be obtained by integrating the PCT with lactate, total bilirubin and APACHII score. Additionally, our results may help to establish a potential screening stool for early detection of DIC induced by septic shock in developing countries.

\section{Declarations}

\section{Ethics approval and consent to participate}

The study protocol was approved by the Second People's Hospital of Shenzhen with ethical Number: 20160115004. Written informed consent was obtained from each participant or their families by telephone before enrolment.

\section{Consent for publication}

All authors reviewed the manuscript and approved the publication.

\section{Availability of data and materials}

The data sets used and/or analyzed during the current study are available from the corresponding author on reasonable request.

\section{Competing interests}

The authors declare that they have no competing interests

\section{Funding}

This study was supported, in part, by grants from Sanming Project of Medicine in Shenzhen (SZSM20162011), the Beijing Nova Program of China (No. Z171100001117113); Shenzhen Science and Technology Innovation Commission (No. JCYJ20170306091335008, JCYJ20190806163603504) and Clinical Research Project of Shenzhen municipal health commission (SZLY2017007).

\section{Authors' contributions}

\# These authors contributed equally to this study

All authors had full access to all the data in the study and take responsibility for the integrity of the data and the accuracy of the data analysis. GF, XSH, XPC, and MW were responsible for study concept and design. $\mathrm{XSH}, \mathrm{MW}, \mathrm{HCZ}$, and JFL were responsible for collecting the data. XSH and MW were responsible for statistical analysis. GF and MW were responsible for drafting the manuscript. 
Acknowledgements

Not applicable

\section{Authors' information}

Correspondence: Wu Ming, boshiyy@126.com; Tel:(+86)075583676149. Department of Critical Care Medicine and Hospital Infection Prevention and Control, the Second People's Hospital of Shenzhen, 3002 Sun gang W Road, Futian District, Shenzhen, 518035, China;

\section{References}

1. Jackson SP, Darbousset R,Schoenwaelder SM. Thromboinflammation: challenges of therapeutically targeting coagulation and other host defense mechanisms. Blood, 2019,133(9):906-918.

2. Engelmann B,Massberg S. Thrombosis as an intravascular effector of innate immunity. Nat Rev Immunol,2013,13(1):34-45.

3. van der Poll T,Herwald $\mathrm{H}$. The coagulation system and its function in early immune defense. Thromb Haemost,2014,112(4):640-648.

4. Jaimes F, De La Rosa G, Morales C, Fortich F, Arango C, Aguirre D,et al. Unfractioned heparin for treatment of sepsis: A randomized clinical trial (The HETRASE Study). Crit Care Med,2009, 37(4):1185-1196.

5. Hayakawa M, Saito S, Uchino S, Yamakawa K, Kudo D, et al. Characteristics, treatments, and outcomes of severe sepsis of 3195 ICU-treated adult patients throughout Japan during 2011-2013. J Intensive Care ,2016,4:44.

6. Taylor FB Jr., Toh CH, Hoots WK, Wada H, Levi M, Scientific Subcommittee on Disseminated Intravascular Coagulation (DIC) of the International Society on Thrombosis and Haemostasis (ISTH). Towards definition, clinical and laboratory criteria, and a scoring system for disseminated intravascular coagulation. Thromb Haemost,2001, 86(5):1327-1330.

7. Angus DC,van der Poll T. Severe sepsis and septic shock. N Engl J Med,2013,369(9):840-851.

8. Yamakawa K, Umemura Y, Hayakawa M, Kudo D, Sanui M, Takahashi H, et al.: Benefit profile of anticoagulant therapy in sepsis: a nationwide multicentre registry in Japan. Crit Care,2016, 20(1):229.

9. Levi M, Toh CH, Thachil J,Watson HG. Guidelines for the diagnosis and management of disseminated intravascular coagulation. British Committee for Standards in Haematology. Br J Haematol,2009,145(1):24-33.

10. Di Nisio M, Baudo F, Cosmi B, D'Angelo A, De Gasperi A, Malato A, Schiavoni M, et al. Italian Society for T,Haemostasis: Diagnosis and treatment of disseminated intravascular coagulation: guidelines of the Italian Society for Haemostasis and Thrombosis (SISET). Thromb Res ,2012,129(5):e177-184. 
11. Wada H, Asakura H, Okamoto K, Iba T, Uchiyama T, Kawasugi K, et al. Expert consensus for the treatment of disseminated intravascular coagulation in Japan. Thromb Res,2010, 125(1):6-11.

12. Wada H, Matsumoto T,Yamashita Y. Diagnosis and treatment of disseminated intravascular coagulation (DIC) according to four DIC guidelines. J Intensive Care,2014, 2(1):15.

13. Iwai K, Uchino S, Endo A, Saito K, Kase Y,Takinami M. Prospective external validation of the new scoring system for disseminated intravascular coagulation by Japanese Association for Acute Medicine (JAAM). Thromb Res,2010, 126(3):217-221.

14. Luo L, Wu Y, Niu T, Han Y, Feng Y, Ding Q, et al. A multicenter, prospective evaluation of the Chinese Society of Thrombosis and Hemostasis Scoring System for disseminated intravascular coagulation. Thromb Res,2019, 173:131-140.

15. Hasegawa D, Nishida K, Hara Y, Kawaji T, Moriyama K, Shimomura Y, et al. Differential effect of lactate in predicting mortality in septic patients with or without disseminated intravascular coagulation: a multicenter, retrospective, observational study. J Intensive Care ,2019,7:2.

16. Patel P, Walborn A, Rondina M, Fareed J,Hoppensteadt D. Markers of Inflammation and Infection in Sepsis and Disseminated Intravascular Coagulation. Clin Appl Thromb Hemost,2019, 25:1076029619843338.

17. Yang X, Cheng X, Tang Y, Qiu X, Wang Z, Fu G, et al. The role of type 1 interferons in Gram-negative bacteria-induced coagulation. Blood, 2020, 135(14):1087-1100.

18. Riedel S. Procalcitonin and the role of biomarkers in the diagnosis and management of sepsis. Diagn Microbiol Infect Dis,2012, 73(3):221-227.

19. Muller B, Becker KL, Schachinger H, Rickenbacher PR, Huber PR, Zimmerli W,et al. Calcitonin precursors are reliable markers of sepsis in a medical intensive care unit. Crit Care Med,2000,28(4):977-983.

20. Wacker C, Prkno A, Brunkhorst FM,Schlattmann P. Procalcitonin as a diagnostic marker for sepsis: a systematic review and meta-analysis. Lancet Infect Dis,2013,13(5):426-435.

21. Samuels JM, Moore HB,Moore EE. Coagulopathy in Severe Sepsis: Interconnectivity of Coagulation and the Immune System. Surg Infect (Larchmt),2018, 19(2):208-215.

22. Seymour CW, Liu VX, Iwashyna TJ, Brunkhorst FM, Rea TD, Scherag A, et al. Assessment of Clinical Criteria for Sepsis: For the Third International Consensus Definitions for Sepsis and Septic Shock (Sepsis-3). JAMA,2016,315(8):762-774.

23. Levi M, Schultz M,van der Poll T. Disseminated intravascular coagulation in infectious disease. Semin Thromb Hemost,2010, 36(4):367-377.

24. Hoppensteadt D, Tsuruta K, Hirman J, Kaul I, Osawa Y,Fareed J. Dysregulation of inflammatory and hemostatic markers in sepsis and suspected disseminated intravascular coagulation. Clin Appl Thromb Hemos,2015,t 21(2):120-127.

25. Fleischmann C, Scherag A, Adhikari NK, Hartog CS, Tsaganos T, Schlattmann P, et al. Reinhart $\mathrm{K}$,International Forum of Acute Care T: Assessment of Global Incidence and Mortality of Hospitaltreated Sepsis. Current Estimates and Limitations. Am J Respir Crit Care Med,2016, 193(3):259-272. 
26. Kumar G, Kumar N, Taneja A, Kaleekal T, Tarima S, McGinley E, et al. Nationwide trends of severe sepsis in the 21st century (2000-2007). Chest,2011,140(5):1223-1231.

27. Martin GS, Mannino DM, Eaton S,Moss M. The epidemiology of sepsis in the United States from 1979 through 2000. N Engl J Med, 2003, 348(16):1546-1554.

28. Gando S, Shiraishi A, Yamakawa K, Ogura H, Saitoh D, Fujishima S, et al. Role of disseminated intravascular coagulation in severe sepsis. Thromb Res,2019, 178:182-188.

29. Wada H, Thachil J, Di Nisio M, Mathew P, Kurosawa S, Gando S, et al. Guidance for diagnosis and treatment of DIC from harmonization of the recommendations from three guidelines. $J$ Thromb Haemost, 2013 Feb 4. doi: 10.1111/jth.12155.

30. Umemura Y, Yamakawa K, Hayakawa M, Hamasaki T, Fujimi S, Japan Septic Disseminated Intravascular Coagulation (J-Septic DIC) study group. Screening itself for disseminated intravascular coagulation may reduce mortality in sepsis: A nationwide multicenter registry in Japan. Thromb Res,2018, 161:60-66.

31. 31.Mei H, Jiang Y, Luo L, Huang R, Su L, Hou M, et al. Evaluation the combined diagnostic value of TAT, PIC, tPAIC, and sTM indisseminated intravascular coagulation: A multi-center prospectiveobservational study.Thromb Res. 2019,173:20-26.

32. Chand N,Sanyal AJ. Sepsis-induced cholestasis. Hepatology,2007, 45(1):230-241.

33. Patel JJ, Taneja A, Niccum D, Kumar G, Jacobs E,Nanchal R. The association of serum bilirubin levels on the outcomes of severe sepsis. J Intensive Care Med,2015, 30(1):23-29.

\section{Tables}

Table 1: Baseline characteristics of non-DIC and DIC patients with sepsis shock 


\begin{tabular}{|c|c|c|c|c|}
\hline Variables & ALL『n=164冈囚 & Non-DIC $\otimes n=106 \rrbracket$ & DIC $₫ n=58 \rrbracket$ & $\begin{array}{l}P \\
\text { value }\end{array}$ \\
\hline Age (years), mean (S.D.) & $68.0(50.8-80.2)$ & $69.5(51.2-81.8)$ & $64.5(46.8-78.0)$ & 0.15 \\
\hline Gender & & & & 0.44 \\
\hline male $\mathrm{N}(\%)$ & 97 & $65 \rrbracket 61.3 \% \rrbracket$ & $32 \rrbracket 55.2 \% \rrbracket$ & \\
\hline Female N (\%) & 67 & $41 \rrbracket 38.7 \% \rrbracket$ & $26 \rrbracket 44.8 \% \rrbracket$ & \\
\hline Infection sources & & & & 0.41 \\
\hline Lungs $N(\%)$ & 52 & $37 \rrbracket 34.9 \% \rrbracket$ & $15 \rrbracket 25.9 \% \rrbracket$ & \\
\hline Urinary tract $\mathrm{N}(\%)$ & 23 & $14 \rrbracket 13.2 \% \bigotimes$ & $9 \rrbracket 15.5 \% \rrbracket$ & \\
\hline Blood N (\%) & 5 & $2 \rrbracket 1.9 \% \rrbracket$ & $3 \rrbracket 5.2 \% \rrbracket$ & \\
\hline Abdominal cavity $\mathrm{N}(\%)$ & 42 & $25 \rrbracket 23.6 \% \rrbracket$ & $17 \rrbracket 29.3 \% \rrbracket$ & \\
\hline Skin and soft tissue N (\%) & 7 & $3 \llbracket 2.8 \% \rrbracket$ & $4 \rrbracket 6.9 \% \rrbracket$ & \\
\hline Two or more $\mathrm{N}(\%)$ & 35 & $25 \rrbracket 23.6 \% \rrbracket$ & $10 \otimes 17.2 \% \rrbracket$ & \\
\hline \multicolumn{5}{|l|}{ Biochemical parameters } \\
\hline WBC $\otimes 1 \times 10^{9} / \mathrm{L} \rrbracket$ & 13.4(7.9-20.2) & $13.4(7.7,20.4)$ & 13.3(8.5,19.9) & 0.81 \\
\hline Hemoglobin (g/L) (IQR) & 109ه92.3-123.0区 & 110.5区93-124.3区 & 106.5区90.8-120区 & 0.35 \\
\hline $\begin{array}{l}\text { Lymphocyte }\left(1 \times 10^{9} / \mathrm{L}\right) \\
\text { (IQR) }\end{array}$ & $0.60 \rrbracket 0.38-1.31 \rrbracket$ & $0.60 \rrbracket 0.36-1.16 \rrbracket$ & $0.63 \rrbracket 0.42-1.33 \rrbracket$ & 0.53 \\
\hline Neutrophil $\left(1 \times 10^{9} / \mathrm{L}\right)(\mathrm{IQR})$ & $11.9(6.8-17.4)$ & $12.4(6.7-17.4)$ & $11.5(8.1-16.9)$ & 0.83 \\
\hline Platelets $\left(1 \times 10^{9} / \mathrm{L}\right)(\mathrm{IQR})$ & 147.0(88.5-213.2) & $\begin{array}{l}172.0(127.2- \\
234.8)\end{array}$ & $76.0(52.0-147.2)$ & $<0.001$ \\
\hline $\mathrm{CRP}(\mathrm{mg} / \mathrm{dl})(\mathrm{IQR})$ & 125.8(71.6-174.8) & $125.0(69.0-174.9)$ & 137.1(85.8-163.0) & 0.79 \\
\hline $\mathrm{PCT}(\mathrm{ng} / \mathrm{ml})(\mathrm{IQR})$ & 20.4凶5.3-102.5区 & $12.6 \rrbracket 2.4-53.3 \rrbracket$ & $54.6 \rrbracket 13.6-200 \rrbracket$ & $<0.001$ \\
\hline Albumin (g/l) (IQR) & $24.5(20.6-28.0)$ & $24.9(20.9-28.9)$ & $22.8(19.6-26.6)$ & 0.18 \\
\hline $\mathrm{Tb}$ (umol/L) (IQR) & 16.0ه9.7-28.6ه & 14.7凶9.4-22.3区 & 21.0ه11.7-42.5邓 & 0.01 \\
\hline $\operatorname{ALT}(\mathrm{U} / \mathrm{L})(\mathrm{IQR})$ & $46.0 \rrbracket 26.3-98 \rrbracket$ & $38.5 \rrbracket 25.0-57.0 \rrbracket$ & $96.5 \rrbracket 39.5-262.3 \rrbracket$ & $<0.001$ \\
\hline AST (U/L) (IQR) & 60.5®31.3-154.8》 & $42.5 \rrbracket 27.0-89.0 \rrbracket$ & $\begin{array}{l}126.0 \otimes 58.8- \\
513.5 \rrbracket\end{array}$ & $<0.001$ \\
\hline Creatinine (umol/L) & 140.4(83.1-239.2) & $119.0(69.8-222.7)$ & $\begin{array}{l}\text { 198.0(108.4- } \\
261.0)\end{array}$ & 0.05 \\
\hline BUN(mmol/l) & $11.2(6.6-16.0)$ & $9.9(5.9-15.3)$ & $13.5(9.0-17.0)$ & 0.17 \\
\hline
\end{tabular}




\begin{tabular}{|c|c|c|c|c|}
\hline Lac (mmol/L) & $3.3(2.6-4.8)$ & $3.0(2.5-3.8)$ & $4.3(2.8-7.5)$ & $<0.001$ \\
\hline $\mathrm{PO}_{2} / \mathrm{FiO}_{2}$ & $\begin{array}{l}237.8(163.5- \\
292.7)\end{array}$ & $\begin{array}{l}229.0(170.5- \\
299.5)\end{array}$ & $\begin{array}{l}249.6(145.2- \\
276.7)\end{array}$ & 0.52 \\
\hline $\mathrm{PT}(\mathrm{s})$ & 15.5(13.6-18.9) & $14.3(12.9-15.9)$ & 19.4(17.1-23.7) & $<0.001$ \\
\hline APTT(s) & $45.0(37.0-60.0)$ & $41.1(35.4-47.3)$ & $59.8(43.8-73.6)$ & $<0.001$ \\
\hline INR & $1.3(1.2-1.6)$ & $1.2(1.1-1.4)$ & $1.7(1.5-2.1)$ & $<0.001$ \\
\hline TT(s) & $19.3(17.6-22.0)$ & $19.0(17.6-21.4)$ & 20.7(17.3-24.5) & $<0.001$ \\
\hline $\mathrm{FIB}(\mathrm{g} / \mathrm{L})$ & $3.4(2.3-4.4)$ & $3.7(2.7-4.7)$ & 2.9(17.1-23.7) & $<0.001$ \\
\hline D-dimer(mg/L) & 8.2(3.5-18.3) & $5.3(3.0-10.5)$ & 18.7(9.7-31.1) & $<0.001$ \\
\hline Mechanical Ventilation & & & & 0.36 \\
\hline yes $N(\%)$ & 91 & $56 \rrbracket 52.8 \% \bigotimes$ & $35 \rrbracket 60.3 \% \rrbracket$ & \\
\hline no $N(\%)$ & 73 & $50 \rrbracket 47.2 \% \rrbracket$ & $23 \llbracket 39.7 \% \rrbracket$ & \\
\hline LOS ICU, median (IQR) (d) & $8.0 \rrbracket 3.0-18.0 \rrbracket$ & $9.0 \rrbracket 4.0-20.0 \rrbracket$ & $4.0 \rrbracket 2.0-13.0 \rrbracket$ & 0.02 \\
\hline APACHE『score & $22.0(16.0-31.0)$ & $20.5(16.0-28.8)$ & 29.5(19.2-34.8) & 0.001 \\
\hline ISTH score & $4.0(3.0-5.0)$ & $3.0(3.0-4.0)$ & $5.0(5.0-6.0$ & $<0.001$ \\
\hline 28-day mortality ( $\mathrm{n} \%$ ) & $72(43.9)$ & $41(38.7)$ & $31(53.4)$ & 0.07 \\
\hline
\end{tabular}

IQR: interquartile range; WBC: White blood cell; CRP: C reactive protein; PCT: procalcitonin; Tb: Total bilirubin; ALT $₫$ Alanine aminotransferase; AST: Aspartate aminotransferase; BUN: Blood urea nitrogen; Lac: Lactic acid; LOS, Length of stay; APACHE-II, Acute Physiology and Chronic Health Evaluation score; ISTH: international society of thrombosis and homeostasis;

Table 2: Risk factors associated with DIC induced by septic shock in univariable and multivariable regression model 


\begin{tabular}{|lrlll|}
\hline Variables & Univariable & & \multicolumn{3}{l|}{ Multivariable } \\
& & & \multicolumn{3}{l|}{ Fully adjusted model } \\
& OR $(95 \% \mathrm{Cl})$ & P value & OR $(95 \% \mathrm{Cl})$ & P value \\
\hline Age (years) & $0.988(0.971,1.004)$ & 0.149 & $0.981(0.956,1.006)$ & 0.128 \\
\hline Male & $1.288(0.673,2.464)$ & 0.444 & $1.491(0.635,3.505)$ & 0.359 \\
\hline PCT (ng/mI囚 & $1.009(1.004,1.013)$ & $<0.001$ & $1.011(1.006,1.016)$ & $<0.001$ \\
\hline Tb (umol/L) & $1.009(1.001,1.017)$ & 0.022 & $1.010(1.001,1.02)$ & 0.035 \\
\hline ALT(U/L) & $1.005(1.002,1.009)$ & 0.003 & $1.000(0.995,1.005)$ & 0.798 \\
\hline AST(U/L) & $1.004(1.001,1.007)$ & 0.004 & $1.002(0.999,1.005)$ & 0.140 \\
\hline Lac (mmol/L) & $1.290(1.126,1.477)$ & $<0.001$ & $1.186(1.005,1.399)$ & 0.044 \\
\hline APACHE score & $1.059(1.021,1.098)$ & 0.002 & $1.046(0.991,1.103)$ & 0.110 \\
\hline & & & & \\
\hline
\end{tabular}

PCT: procalcitonin; Tb: Total bilirubin; ALT囚Alanine aminotransferase; AST: Aspartate aminotransferase; Lac: Lactic acid; APACHE-II, Acute Physiology and Chronic Health Evaluation score;

Fully adjusted model: Age, Gender

\section{Table30Predicting DIC induced by septic shock with ROC curve}

\begin{tabular}{|c|c|c|c|c|}
\hline Variables & $\operatorname{AUC}(95 \% \mathrm{Cl})$ & Cut-off value & Sensitivity, specificity & $P$ value \\
\hline РСТ $(\mathrm{ng} / \mathrm{ml})$ & $0.701(0.619-0.784)$ & 42.1 & $60.34 \% \quad 72.64 \%$ & $<0.001$ \\
\hline $\operatorname{Lac}(\mathrm{mmol} / \mathrm{L})$ & $0.669(0.579-0.759)$ & 4.2 & $51.70 \%, \quad 77.40 \%$ & $<0.001$ \\
\hline $\mathrm{Tb}$ (umol/L) & $0.621(0.525-0.717)$ & 31.8 & $37.93 \%, \quad 86.79 \%$ & 0.010 \\
\hline APACHEX & $0.643(0.550-0.736)$ & 28.5 & $53.45 \%, \quad 74.53 \%$ & 0.002 \\
\hline Lact+ APACHE $₫+T b$ & $0.706(0.630-0.774)$ & $\mathrm{N} / \mathrm{A}$ & $65.5 \% \quad 80.20 \%$ & $<0.001$ \\
\hline PCT+Lact+ APACHE + Tb & $0.801 \rrbracket 0.732-0.871 \rrbracket$ & $\mathrm{N} / \mathrm{A}$ & $74.1 \%$ & $<0.001$ \\
\hline
\end{tabular}

PCT: procalcitonin; Lact: Lactic acid; Tb: Total bilirubin; APACHE-II, Acute Physiology and Chronic Health Evaluation score; 
2154 patients admitted to University Hospital between January 2015 and December 2018

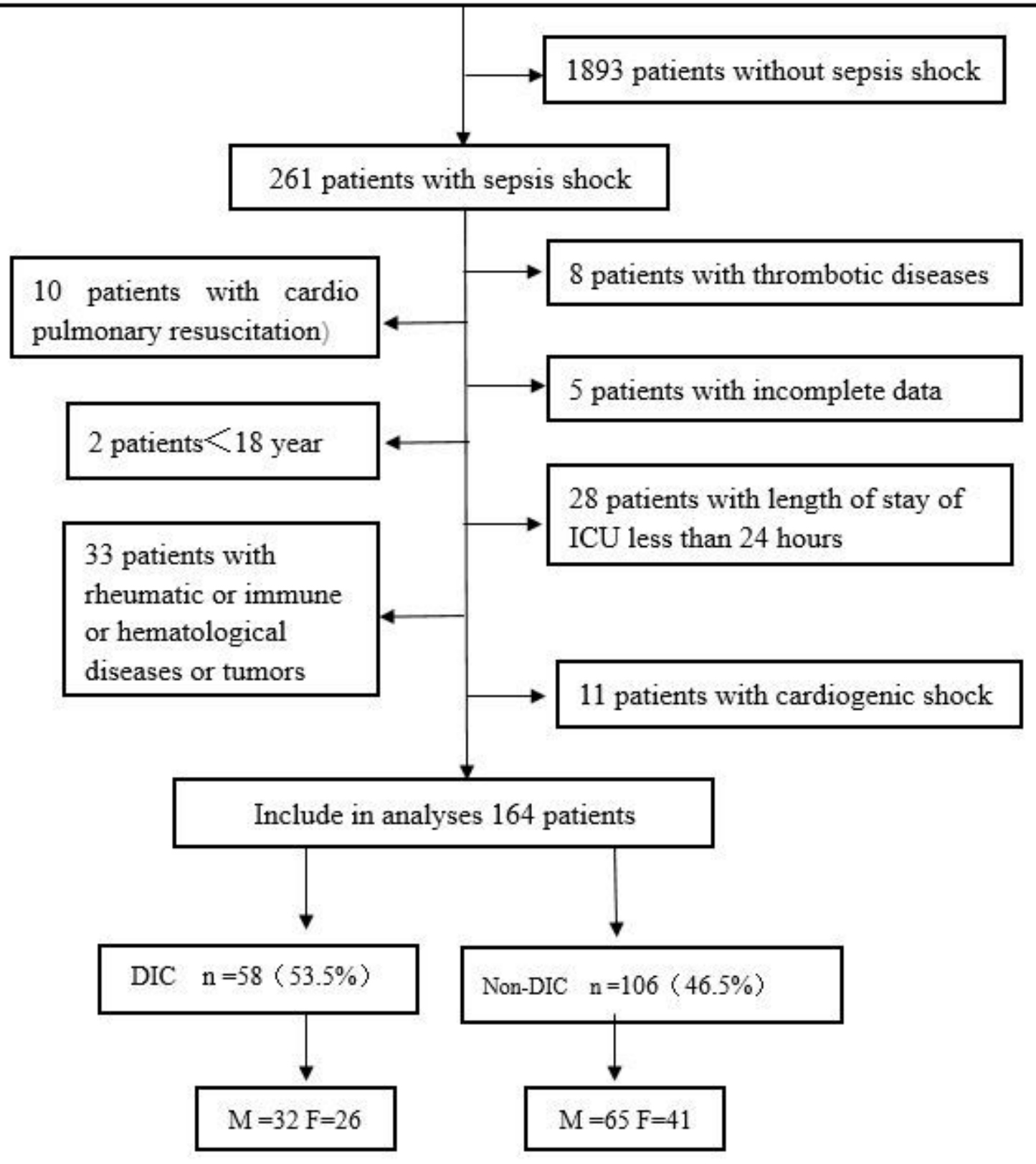

Figure 1

Flow diagram of study subjects 


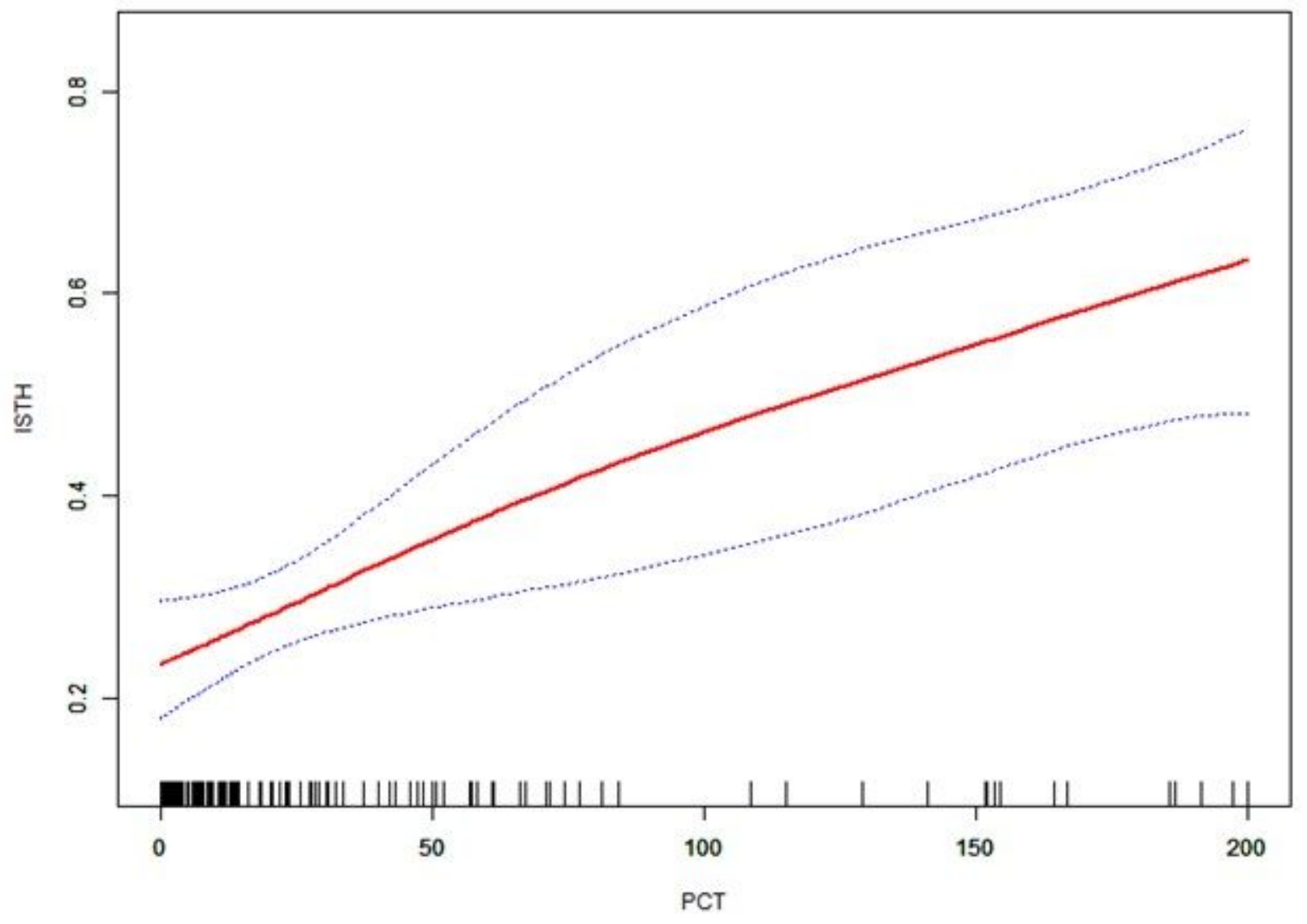

Figure 2

The relationship between PCT and DIC (ISTH) by smooth curve fitting
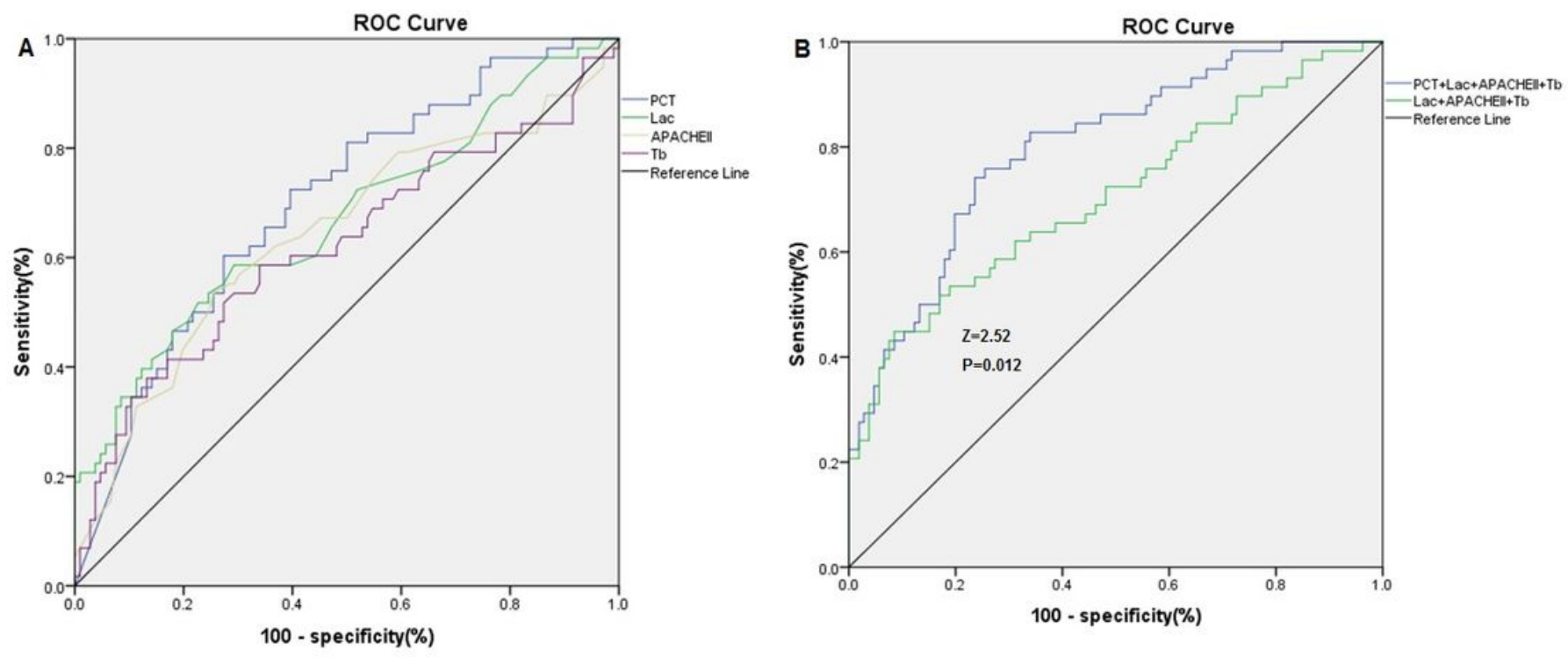

Figure 3 
The ROC curve of PCT, Lac, Tb and APACHEll scores 